\title{
Is Lockdown Effective in Limiting SARS-CoV-2 Epidemic Progression?-a Cross-Country Comparative Evaluation Using Epidemiokinetic Tools
}

\author{
Bruno Mégarbane, MD, $P h D^{1,2}$ (D) Fanchon Bourasset, PharmD, $P h D^{2,3}$, and \\ Jean-Michel Scherrmann, PharmD, $P h D^{2,3}$
}

'Department of Medical and Toxicological Critical Care, Lariboisière Hospital, Federation of Toxicology, APHP, Paris, France; ${ }^{2}$ University of Paris, INSERM UMRS-1 144, Paris, France; ${ }^{3}$ Laboratory of Pharmacokinetics, Faculty of Pharmacy, Paris, France.

BACKGROUND: To date, the risk/benefit balance of lockdown in controlling severe acute respiratory syndrome coronavirus-2 (SARS-CoV-2) epidemic is controversial. OBJECTIVE: We aimed to investigate the effectiveness of lockdown on SARS-CoV-2 epidemic progression in nine different countries (New Zealand, France, Spain, Germany, the Netherlands, Italy, the UK, Sweden, and the USA). DESIGN: We conducted a cross-country comparative evaluation using a susceptible-infected-recovered (SIR)based model completed with pharmacokinetic approaches.

MAIN MEASURES: The rate of new daily SARS-CoV-2 cases in the nine countries was calculated from the World Health Organization's published data. Using a SIR-based model, we determined the infection $(\beta)$ and recovery $(\gamma)$ rate constants; their corresponding half-lives $\left(t_{1 / 2 \beta}\right.$ and $\left.t_{1 / 2 \gamma}\right)$; the basic reproduction numbers $\left(R_{0}\right.$ as $\left.\beta / \gamma\right)$; the rates of susceptible $\mathrm{S}(\mathrm{t})$, infected $\mathrm{I}(\mathrm{t})$, and recovered $\mathrm{R}(\mathrm{t})$ compartments; and the effectiveness of lockdown. Since this approach requires the epidemic termination to build the (I) compartment, we determined S(t) at an early epidemic stage using simple linear regressions.

KEY RESULTS: In New Zealand, France, Spain, Germany, the Netherlands, Italy, and the UK, early-onset stayat-home orders and restrictions followed by gradual deconfinement allowed rapid reduction in SARS-CoV-2infected individuals $\left(t_{1 / 2 \beta} \leq 14\right.$ days) with $R_{0} \leq 1.5$ and rapid recovery $\left(t_{1 / 2 \gamma} \leq 18\right.$ days). By contrast, in Sweden (no lockdown) and the USA (heterogeneous statedependent lockdown followed by abrupt deconfinement scenarios), a prolonged plateau of SARS-CoV-2-infected individuals (terminal $t_{1 / 2 \beta}$ of 23 and 40 days, respectively) with elevated $R_{0}$ ( 4.9 and 4.4 , respectively) and nonending recovery (terminal $t_{1 / 2 \gamma}$ of 112 and 179 days, respectively) was observed.

CONCLUSIONS: Early-onset lockdown with gradual deconfinement allowed shortening the SARS-CoV-2 epidemic and reducing contaminations. Lockdown should be considered as an effective public health intervention to halt epidemic progression.

Bruno Mégarbane and Fanchon Bourasset contributed equally to this work.

Received August 3, 2020

Accepted November 8, 2020

Published online January 13, 2021
KEY WORDS: COVID-19; epidemic; lockdown; modeling; pharmacokinetics; SARS-CoV-2.

J Gen Intern Med 36(3):746-52

DOI: $10.1007 /$ s11606-020-06345-5

(C) Society of General Internal Medicine 2020

\section{INTRODUCTION}

Starting in fall 2019 in Wuhan, China, the severe acute respiratory syndrome coronavirus-2 (SARS-CoV-2) pandemic has rapidly spread worldwide. ${ }^{1}$ In the absence of effective antiviral therapy, social distancing, handwashing, and face covers were promoted to control SARS-CoV-2 spread. ${ }^{2}$ However, due to increasing severe coronavirus disease-2019 (COVID-19) presentations and critical care bed saturation, almost all governments decided on lockdown. ${ }^{3}$ Lockdown, variably including stay-at-home orders, workplace restrictions, and school and venue closures, was applied on an unprecedented scale during 30-90 days then progressively released following reduction in SARS-CoV-2 spread.

The risk/benefit balance of restrictions and optimal lockdown modalities to control SARS-CoV-2 epidemic are controversial. $^{4-6}$ As more data become available, predictive models are updated, limiting reasonable accuracy to shortterm projections and empirical fitting of data trends. ${ }^{7,8}$ Whereas daily numbers of COVID-19-related fatalities, critical cases, and hospital admissions are trustworthy, numbers of daily new contaminations (I), useful for monitoring SARS-CoV-2 spread, are underestimated due to limited testing.

Modeling used to guide non-pharmaceutical interventions including lockdown policy is based on the compartmental susceptible-infected-recovered (SIR) model. ${ }^{7,8}$ Introduced in 1927, the SIR model aims to predict the number of individuals who are susceptible to the infection, are actively infected, or have recovered from infection at any given time. ${ }^{9}$ According to their disease status, the individuals of the studied population are divided into three mutually exclusive groups determining the susceptible, infected, and recovered compartments. A susceptible individual, counted in the $\mathrm{S}$ compartment, may become infected following a contact with another infected individual. He is assumed to be contagious and starts to be counted 
in the I compartment until moving to a non-contagious stage named recovery (that may include effective isolation or death). Thereafter, he is counted in the R compartment.

Interestingly, the SIR and derived more stochastic models share similarities with compartmental pharmacokinetic models. The SIR model structure resembles a closed threecompartmental pharmacokinetic catenary model describing drug absorption and elimination processes as the sum of an input and output function. ${ }^{8}$ If translated to pandemic kinetics, the input function could represent the epidemic progression phase or "infection rate" and the output function its regression phase or "recovery rate". We considered that overall kinetics could be assessed by considering (I) as the best endpoint to measure the disease progression rate despite restricted testing.

Although many models of infectious disease transmission in general and of SARS-CoV-2 in particular continue to utilize the basic framework of SIR, they typically incorporate additional information into their estimation of the three main SIR parameters. Our purpose was to propose a simpler model, based on fewer parameters, able to provide similarly and early accurate predictions. We aimed to compare lockdownattributed effects on SARS-CoV-2 epidemic progression in nine countries with various lockdown scenarios. Applying the analogous tools of pharmacokinetics to quantify the transmission kinetics of SARS-CoV-2 allows estimating the time-toreach the plateau and epidemic length in each country. These parameters may prove to be useful for health authorities to rapidly determine the effectiveness of the undertaken restrictions and inform the population on their expected duration.

\section{METHODS}

\section{Design and Setting}

For each country, we collected 7-day interval (I) from 23 Feb 2020 to 14 Jun 2020 published by the World Health Organization (WHO; https://covid19.who.int/) and calculated the daily case rate as follows:

$\mathrm{dI} / \mathrm{dt}=\frac{\sum_{\text {day } n}^{\text {day } n+7} I}{7}$.

We plotted I $(\mathrm{t})$ versus time using the excretion-rate pharmacokinetic method at mid-point, ${ }^{9}$ developed to assess the urinary drug excretion rate by considering that successive drug amounts $\left(X_{\mathrm{u}}\right)$ excreted within defined time intervals can be plotted versus time using the excretion rate $\left(\mathrm{d} X_{\mathrm{u}} / \mathrm{dt}\right)$ at the midpoint of this time interval. This method allowed averaging the effect of sample collection variability, as observed in declared (I) with underestimated values each weekend corrected on the first-days of the following week.

We fitted $I(t)$ using equations $(1+2)$ and used the estimated parameters to simulate the kinetics in the susceptible $(\mathrm{S}(\mathrm{t}))$ and recovered $(\mathrm{R}(\mathrm{t})$ ) compartments using equations (3+4) (Fig. 1). Aware that (I) could be only determined by this approach once the epidemic ends, we used simple linear regressions to build
$\mathrm{S}(\mathrm{t})$ at an early stage of the epidemic. We calculated the mean $\pm \mathrm{SEM}$ of $\mathrm{S}=\mathrm{Ic}_{(\mathrm{day} n)}-\mathrm{Ic}_{(\text {dayn-1) }} / \mathrm{Ic}_{(\text {dayn-1) }} \times 100$ each 7-day period, where Ic represented cumulative (I). S(t) was fitted versus the mid-point time interval.

Since no consensual definition of lockdown exists and since its exact content varied between the countries and along its application time, we considered the stay-at-home order decided by the government as the start of the studied exposure and the decision of stopping the main policy measures as the end of exposure.

\section{Analysis}

Modeling was performed using Phoenix64-WinNonlin ${ }^{\mathrm{TM}}$ (Certara, USA). All parameters of the models were estimated and not fixed to avoid any over-determination of the models. We determined the maximum rate of new cases $\left(I_{\max }\right)$, the maximum $\mathrm{S}$ at $t=0\left(\mathrm{~S}_{0}\right)$, the infection $(\beta)$ and recovery $(\gamma)$ rate constants, the corresponding half-lives $\left(t_{1 / 2 \beta}=\ln 2 / \beta\right.$ and $\left.t_{1 / 2 \gamma}=\ln 2 / \gamma\right)$, and the basic reproduction number $\left(R_{0}=\beta / \gamma\right){ }^{8}$ We also determined the lag-time (Tlag) as the time difference between the lockdown start-time and the input phase end-time $\left(t_{\mathrm{i}}\right)$. Criteria to evaluate the modeling included (1) fits between observed and predicted data; (2) Akaike criteria, lowered as possible to minimize the gap between estimated and observed data; (3) coefficients of variation $<30 \%$; and (4) weighted residuals versus time plot that must be randomized above and below zero.

\section{RESULTS}

Using the WHO's published observed (I) data, we modeled $I(t)$ in nine different countries (Fig. 2). We build $S(t)$ using simple linear regressions (Fig. 3). Using these estimated parameters, we simulated $\mathrm{S}(\mathrm{t}), \mathrm{I}(\mathrm{t})$, and $\mathrm{R}(\mathrm{t})$ curves of our SIRbased model (Fig. 4). The kinetic parameters and model adequacy criteria with the observed data are presented in Table 1. All criteria were satisfactory, with the majority of the coefficients of variation below or at around $30 \%$ and correlation coefficients greater than 0.9 for each modeling, showing a satisfactory congruence of the model with the observed data.

Based on our models, two groups of countries could be distinguished. Sweden (no lockdown) and the USA (variable lockdown abruptly ended) (named as group 1) exhibited a prolonged plateau of infections, (terminal $t_{1 / 2 \beta}, 23$ and 40 days), non-ending recovery $\left(t_{1 / 2 \gamma}, 112\right.$ and 179 days), and $R_{0}$ of 4.9 and 4.6, respectively. All other countries (named as group 2) showed rapid decrease in infection $\left(t_{1 / 2 \beta} \leq 14\right.$ days), accelerated recovery $\left(t_{1 / 2 \gamma} \leq 18\right.$ days), and $R_{0} \leq 1.5$. In group 2 , New Zealand, France, Spain, and Germany with early-onset and progressively ended lockdown showed Tlag $\leq 15$ days and $t_{\mathrm{i}} \leq 21$ days, whereas the UK, Italy, and the Netherlands (with delayed, area-dependent, and loose lockdowns, respectively) exhibited Tlag $\geq 20$ days and $t_{\mathrm{i}} \geq 30$ days (Fig. 2). 


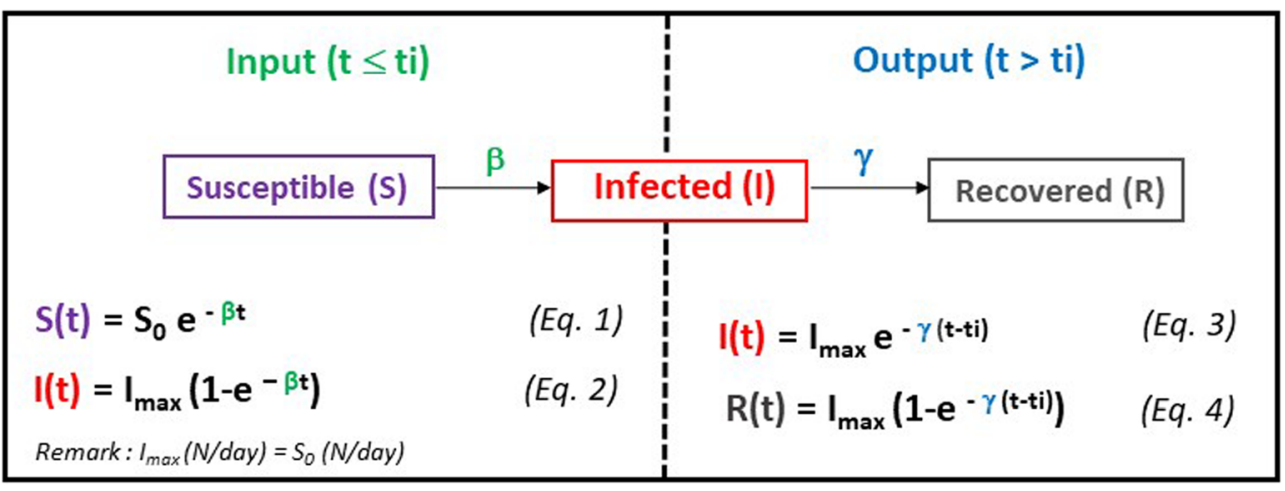

Figure 1 The epidemiokinetic models used to calculate the parameters of the susceptible-infected-recovered (SIR)-based model adapted from Tolles and Luong (2020). During the input phase, susceptible individuals (S) become infected (I). The infection rate constant $(\beta)$ drives the input in the infected compartment. During the output phase, infected patients recover $(\mathbf{R})$. The recovery rate constant $(\gamma)$ drives the output from the infected compartment. I(t) (N/day) is fitted from the observed data (see Fig. 2a). S(t) can be fitted from the observed data (S given in \%; see Fig.

$2 b$ ) or simulated by using Eq. (3) and parameters $\left(S_{0}(N /\right.$ day) and $\beta)$ estimated by fitting $I(t)$. $R(t)$ can be simulated by using Eq. (4) and parameters estimated by fitting $I(t)$ from the observed data (see Fig. 2a).

\section{DISCUSSION}

Our findings support the effectiveness of lockdown in reducing (I) and shortening the SARS-CoV-2 epidemic.

Lockdown-attributed effects on SARS-CoV-2 epidemic regression can be satisfactorily modeled using pharmacokinetic principles. Our affordable epidemiokinetic method relies on the predictive power of the input function as a surveillance tool. Although underestimated due to limited testing, (I) represents an actual sample allowing the confident quantifying of SARS-CoV-2 spread irrespective of the country policy. We a

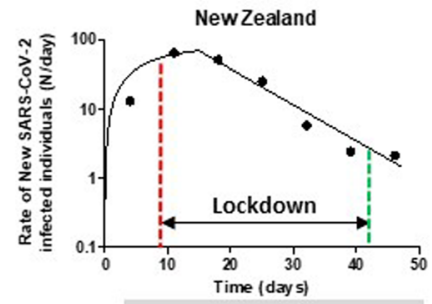

$t_{1 / 2 \gamma}=6$ days

d $\quad t=15$ days, Tlag $=6$ days

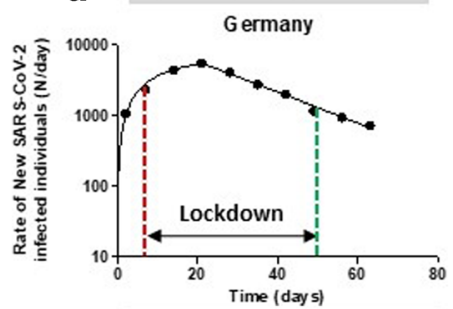

$t_{1 / 2 \gamma}=14$ days

g

$\mathrm{t}_{\mathrm{i}}=21$ days, Tlag $=14$ days

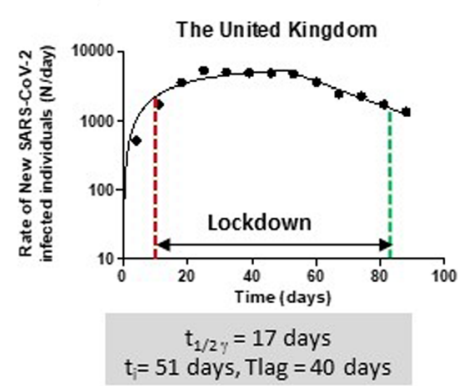

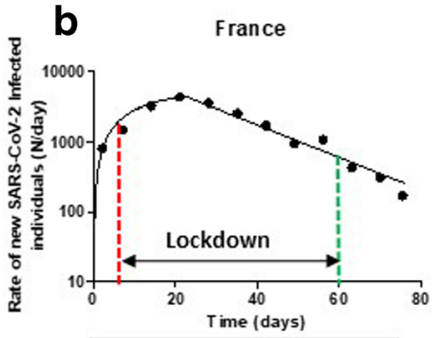

$t_{1 / 2 y}=13$ days $t_{i}=22$ days, $\operatorname{Tlag}=15$ days

The Netherlands

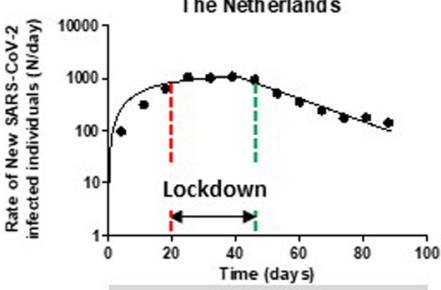

$t_{1 / 2 \gamma}=14$ days $\mathrm{t}_{\mathrm{i}}=41$ days, Tlag $=23$ days

h

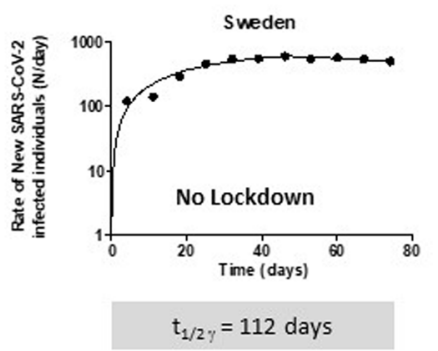

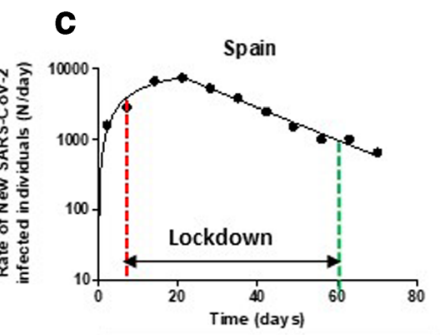
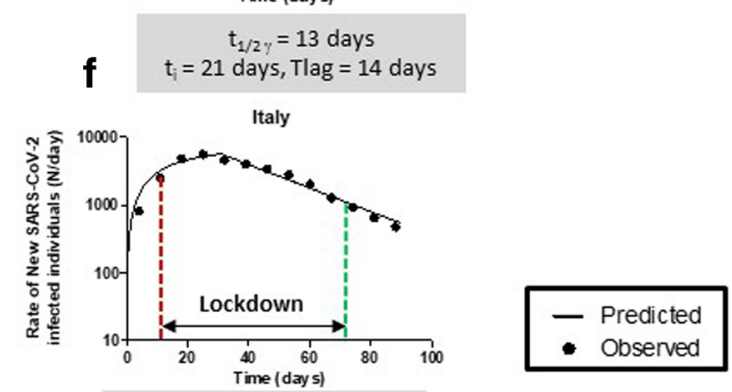

$t_{1 / 2 \%}=18$ days $\mathrm{t}_{\mathrm{i}}=31$ days, Tlag $=20$ days

i

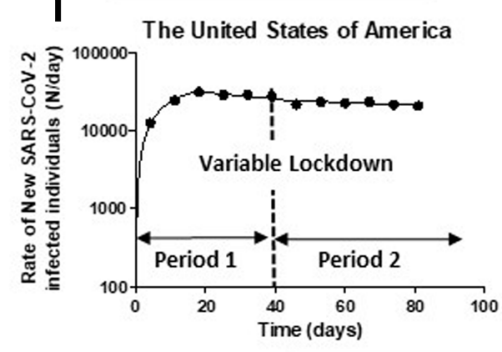

$t_{1 / 21_{1}}=69$ days $\quad t_{1 / 2 \% 2}=179$ days

Figure 2 Rates of new SARS-CoV-2-infected individuals (I(t), N/day) fitted from the observed data in New Zealand (a), France (b), Spain (c), Germany (d), the Netherlands (e), Italy (f), the UK (g), Sweden (h), and the USA (i). The observed data were collected from 23 Feb 2020 to 14 Jun 2020, from the World Health Organization. 

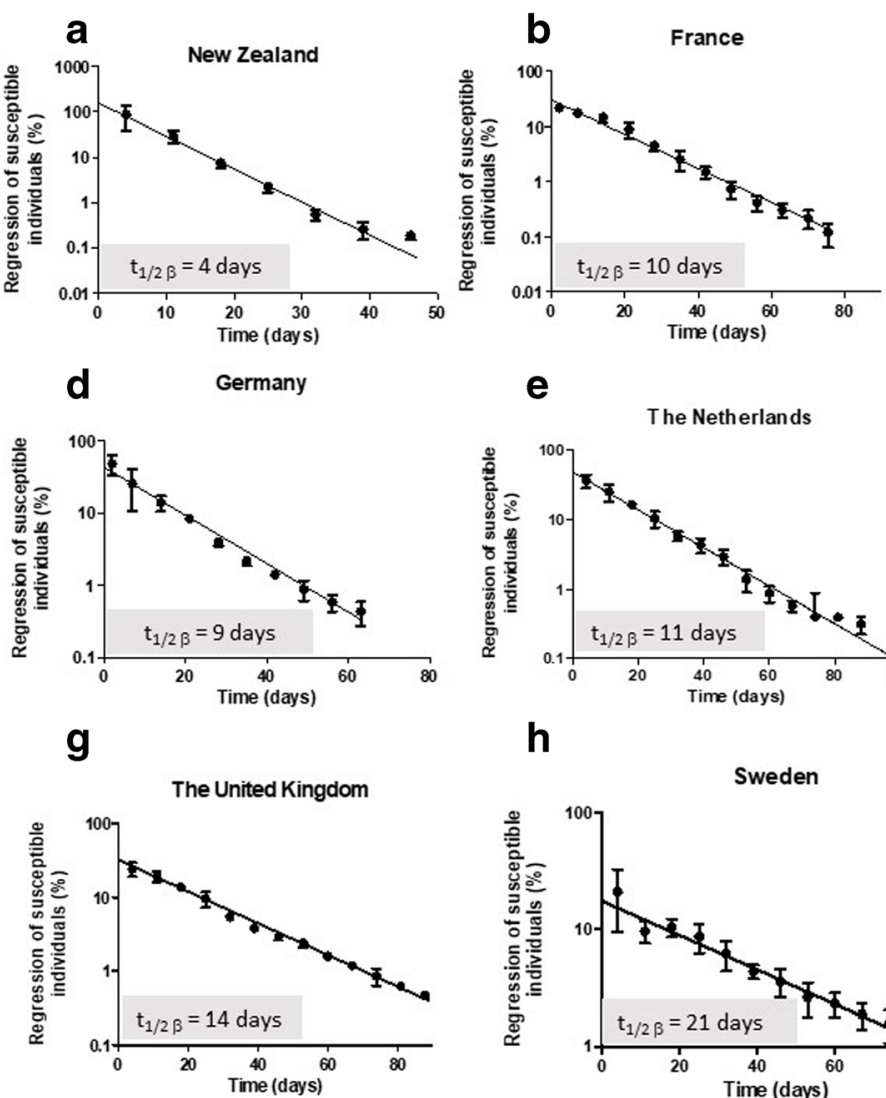

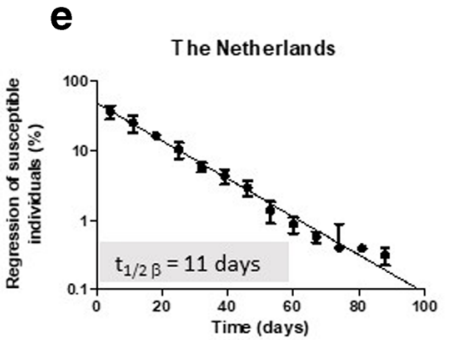

h

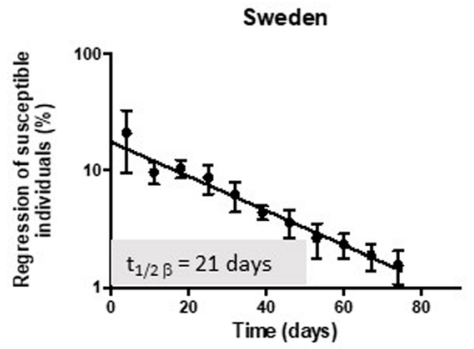

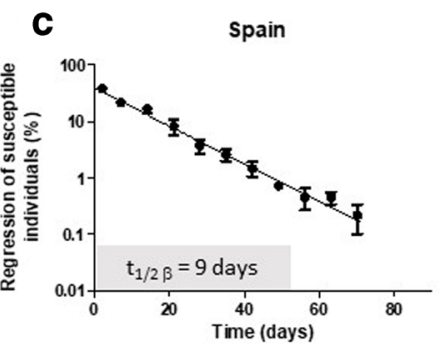

f

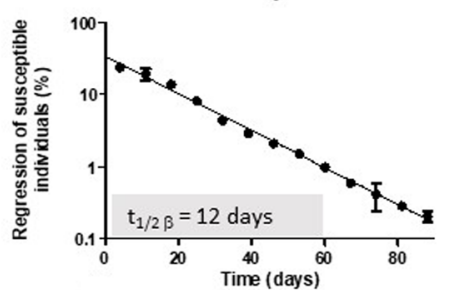

i

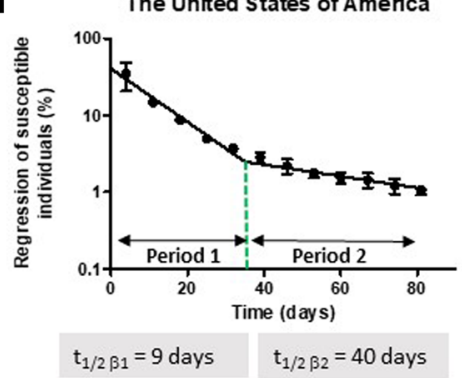

Figure 3 Rates of regression of SARS-CoV-2-susceptible individuals fitted from the observed data in New Zealand (a), France (b), Spain (c), Germany (d), the Netherlands (e), Italy (f), the UK (g), Sweden (h), and the USA (i). The observed data were collected from 23 Feb 2020 to 14 Jun 2020, from the World Health Organization.

used (I) as epidemic progression rate rather than an absolute dimension marker as done in epidemic surveys. This marker allows fitting $\mathrm{I}(\mathrm{t})$, estimating $\beta$ and $\gamma$, and thereafter simulating $\mathrm{S}(\mathrm{t})$ and $\mathrm{R}(\mathrm{t})$ to build the SIR-based model. Our approach's strength was to allow estimating $\beta$ and $\mathrm{I}_{\max }$ using simple linear regressions. Hence, only three points (i.e., 10 days if a 3day interval collection period is chosen) would be necessary. Our $\beta$ estimates by fitting $I(t)$ or $S(t)$ were similar (Table 1). Compared with the sophisticated susceptibleexposed-infectious-recovered (SEIR) model, ${ }^{11}$ our method allowed obtaining similar $\gamma$ estimates for Spain $(0.05$ versus $0.04 \mathrm{day}^{-1}$ ) and the second period in the USA (0.004 versus 0.005 day $\left.^{-1}\right)$. Our approach points to some universality, suggesting that simple mean-field models are helpful to evaluate epidemic kinetics and lockdownattributed effects.

Government-imposed social distancing was shown to reduce the daily case growth rate over time ${ }^{12}$ and presumed to decrease $R_{0}$ mainly by reducing $\beta{ }^{8}$ Our findings supported that coherent lockdown strategies divided $t_{1 / 2 \gamma}$ by $\sim 6.2$-fold and $R_{0}$ by $\sim 3.3$-fold. We confirmed that lockdown alters interindividual SARS-CoV-2 transmission but showed that $R_{0}$ is preferentially steered by $\gamma$ than $\beta(\sim 6.2$ - and $\sim 1.5$-fold decrease in Sweden versus group 2 countries, respectively). Interestingly, despite its heterogeneity, lockdown in the USA resulted first in a $\sim 2.7$-fold and $\sim 1$.6-fold decrease of $t_{1 / 2 \beta}$ and $t_{1 / 2 \gamma}$, respectively, in comparison with Sweden, suggesting that restrictions were effective. However, as suggested, ${ }^{11}$ the abrupt deconfinement scenarios adopted later lead to $\mathrm{a} \sim 4.8$ fold and $\sim 2$.6-fold increase in $t_{1 / 2 \beta}$ and $t_{1 / 2 \gamma}$, respectively, in comparison to the initial period with a pattern similar to Sweden, which deliberately chose a non-lockdown strategy. Comparing pre-order with post-order slopes, US state-level stay-at-home orders were shown to reduce confirmed case rates. ${ }^{13}$ However, estimated cases increased in border counties in Iowa without stay-at-home order compared with Illinois with stay-at-home order. ${ }^{14}$ Consequently, re-ascension in (I) since mid-June could be expected as actually observed in the USA and several other countries worldwide.

Stringency of government responses to SARS-CoV-2 epidemic (scale, 1-100) did not predict their appropriateness or effectiveness. ${ }^{10}$ Here, shorter Tlag and $t_{\mathrm{i}}$, attenuated $\mathrm{I}_{\max }$, and shortened epidemic plateau duration were observed in countries with optimal restriction strategies, especially in New Zealand with strict border control and where the lockdown was started $\leq 1$ month after the first SARS-CoV-2-infected case, whereas it started $\sim 1.5$ months after the first case in France, Germany, Spain, Italy, and the Netherlands and $\sim 2$ months after the first case in the UK and USA. Our data support that early-onset lockdown with sufficient duration 

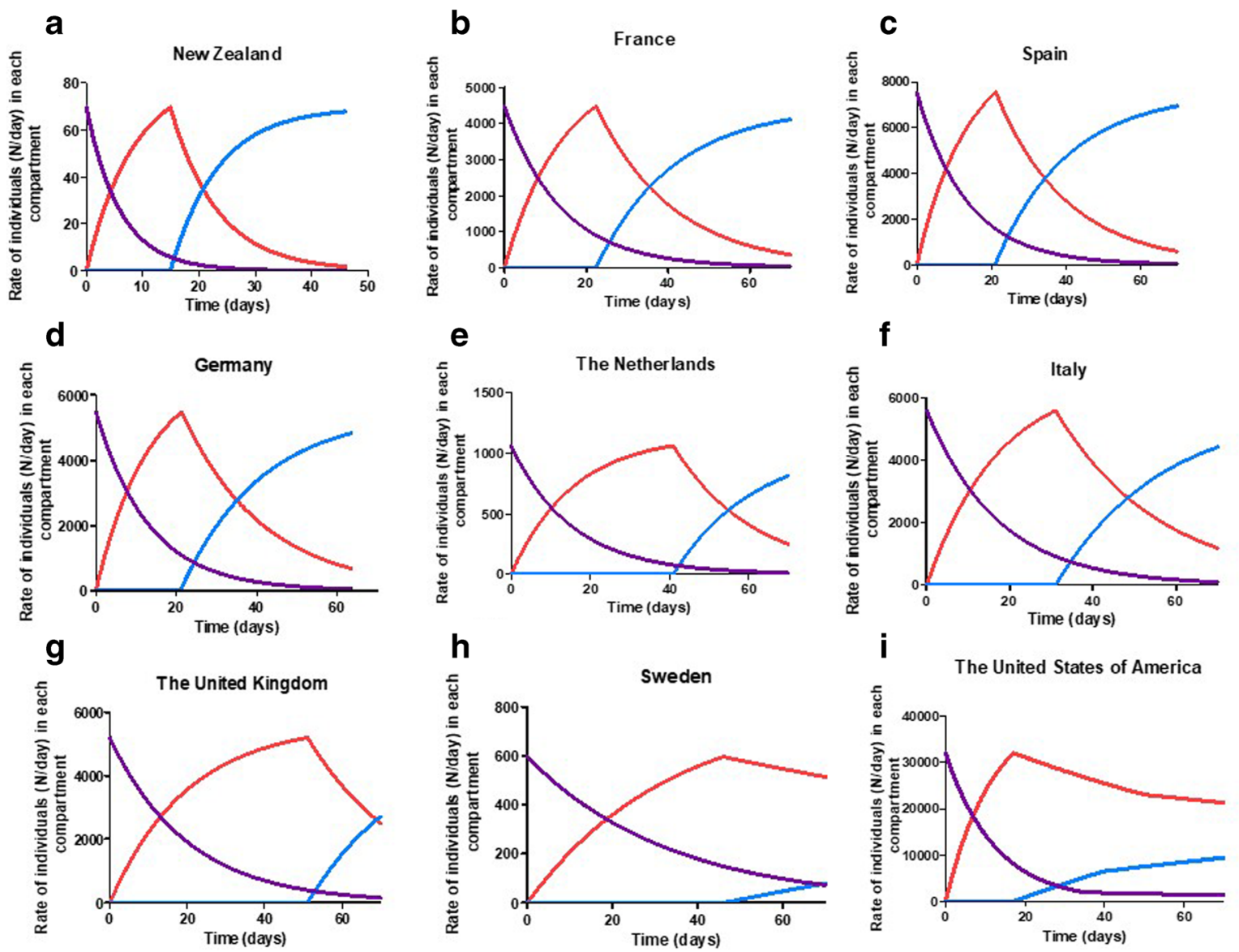

Figure 4 Simulated S(t), I(t), and R(t) curves of our susceptible-infected-recovered (SIR)-based model (2C) in New Zealand (a), France (b), Spain (c), Germany (d), the Netherlands (e), Italy (f), the UK (g), Sweden (h), and the USA (i).

and progressive ending are the key determinants of effectiveness, especially since SARS-CoV-2 contagion lasts 14-20 days. Our data shows that early-onset lockdown was associated with reduced $R_{0}$ especially in New Zealand where $R_{0}$ was $\sim 1$, suggesting that lockdown was efficient to totally remove the epidemic from the population. Interestingly, the more recent figures of SARS-CoV-2 pandemic (October 2020) confirmed that New Zealand is also the unique country among those studied not to experience a second wave. Our findings suggest that optimal lockdown may prevent hospital saturation and limit fatalities. Stay-at-home orders effectively deviated cumulative hospitalizations for COVID-19 from their projected bestfit growth rates. ${ }^{15}$

By analogy to pharmacology in which it is possible to calculate both absorption and elimination half-lives, our method allows estimating the time-to-reach the plateau and epidemic length in each country (e.g., 3.3-fold $t_{1 / 2 \beta}$ and $t_{1 / 2 \gamma}$, corresponding to $\sim 90 \%$ of the case accumulation and epidemic regression, respectively). Hence, these times are prolonged in group 1 versus group 2 countries (i.e., $\sim 100$ days versus 33 days and $\sim 1$ year versus $\sim 38$ days, respectively). However, caution is requested due to the tremendous amount of uncertainty surrounding what we do and do not know about this virus and since the number of susceptible people in the population is unknown and may account for uncertainty of the model. Additional conditions may also influence the epidemic progression including early use of possibly effective treatments, natural herd immunity, and population susceptibility.

Our study has significant limitations. Our model accounts for smooth short-term variation in contamination reporting (e.g., for weekend-related delays), but it may less account for larger sources of variation between and within countries and over time. However, since focused on the contamination progression (slope), our approach is mildly dependent on the exact range of contaminations on condition that testing was performed in a similar manner during the whole study period in one given country. Extremely large variations have been observed between countries as well as within countries and over time in the intent of their lockdown policies, and the fidelity of implementation/ adherence by their respective residents (e.g., enforceable/ enforced mandates versus recommendations). There were also tremendous variations in other strategies intended to reduce transmission such as universal facemask wearing, quality of contract tracing, and availability of temporary housing for isolation and quarantine. We acknowledge that our simplified modeling of SARS-CoV-2 transmission dynamics did not account for all variations that are mandatory to be definitively useful for policy and practice. We also acknowledge that we did not provide direct comparisons between our simplified model and alternative more complex models regarding the relevance to 


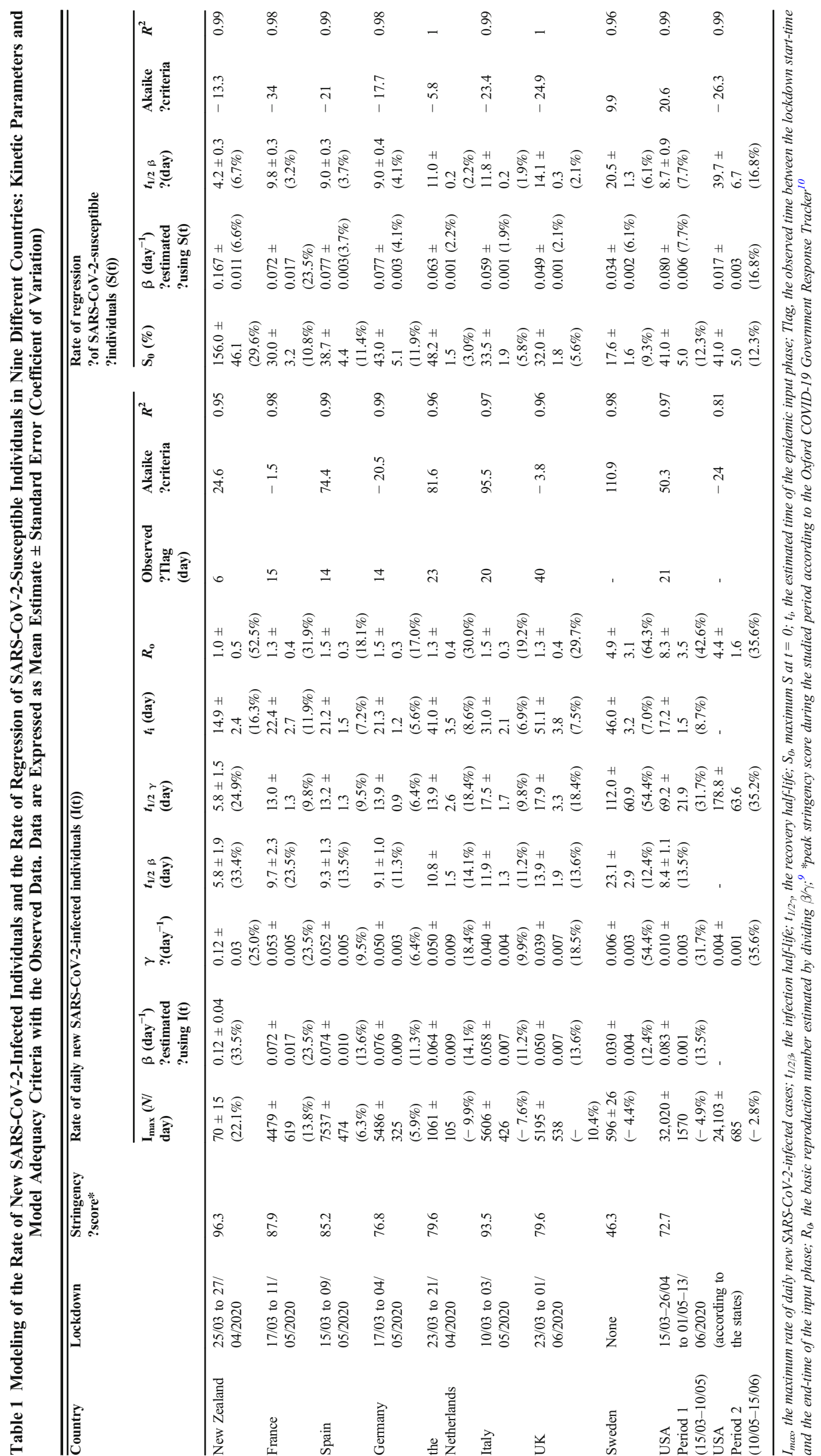


real-world clinical or policy decisions. However, different outputs from our model (e.g., the estimated time-to-reach plateau or the epidemic length) could be used to inform a policy decision. Health authorities' decisions should almost certainly depend not only on the derivative/marginal/relative changes in the rate of transmission but also on the magnitude of transmission on an absolute scale, as possibly provided by our outputs. Noteworthy, interpreting the global transmission dynamics in the USA may be simplistic since many if not most decisions are (and should) be taken at the level of each single state. Finally, the main question to be asked of lockdown policy effectiveness is not categorical (does it work or not?) but rather a suite of more nuanced questions related to the marginal contributions to transmission reduction of various strategies and their combinations, supporting our cross-country comparative study design.

To conclude, SARS-CoV-2 epidemic regression is well described by our epidemiokinetic approach. Lockdown effectiveness to reduce the infection growth rate and shorten the epidemic is better predicted by its early onset and progressive ending than its stringency. However, the optimal lockdown strategy able to reduce demand on healthcare utilization and fatalities remains to be determined.

Acknowledgments: The authors would like to thank Mrs. Alison Good (Scotland, UK) for her helpful review of the manuscript.

Corresponding Author: Bruno Mégarbane, MD, PhD; Department of Medical and Toxicological Critical Care, Lariboisière Hospital, Federation of Toxicology, APHP, Paris, France

(e-mail: bruno.megarbane@lrb.aphp.fr).

Author Contributions Concept and design (all three authors); acquisition, analysis, or interpretation of data (all three authors); drafting of the manuscript (Mégarbane, Bourasset); critical revision of the manuscript for important intellectual content (all three authors).

Funding This investigation, analysis, and manuscript preparation were completed as part of official duties at the university hospital.

Data Availability Dr Mégarbane had full access to all of the data in the study and takes responsibility for data integrity and data analysis accuracy. Data are available from the corresponding author on reasonable request.

\section{Compliance with Ethical Standards:}

Not applicable.

Conflict of Interest: The authors declare that they do not have a conflict of interest.

\section{REFERENCES}

1. Wu Z, McGoogan JM. Characteristics of and Important Lessons From the Coronavirus Disease 2019 (COVID-19) Outbreak in China: Summary of a Report of 72314 Cases From the Chinese Center for Disease Control and Prevention. JAMA. 2020. doi:https://doi.org/10.1001/jama.2020. 2648.

2. Chu DK, Akl EA, Duda S, Solo K, Yaacoub S, Schünemann HJ. Physical distancing, face masks, and eye protection to prevent person-toperson transmission of SARS-CoV-2 and COVID-19: a systematic review and meta-analysis. Lancet. 2020;395:1973-1987.

3. Hsiang S, Allen D, Annan-Phan S, Bell K, Bolliger I, Chong T, et al. The effect of large-scale anti-contagion policies on the COVID-19 pandemic. Nature. 2020. doi:https://doi.org/10.1038/s41586-0202404-8.

4. Ferguson NM, Laydon D, Nedjati-Gilani G, Imai N, Ainslie K, Baguelin $\mathbf{M}$ et al. Impact of non-pharmaceutical interventions (NPIs) to reduce COVID-19 mortality and healthcare demand. Tech Rep, Imperial College London, 2020. Available from: https://www.imperial.ac.uk/media/imperial-college/medicine/sph/ide/gida-fellowships/Imperial-CollegeCOVID19-NPI-modelling-16-03-2020.pdf (29 July 2020, date last accessed).

5. Lewnard JA, Lo NC. Scientific and ethical basis for social-distancing interventions against COVID-19. Lancet Infect Dis. 2020;20:631-633.

6. Savulescu J, Persson I, Wilkinson D. Utilitarianism and the Pandemic. Bioethics. 2020. doi:https://doi.org/10.1111/bioe.12771.

7. Jewell NP, Lewnard JA, Jewell BL. Predictive Mathematical Models of the COVID-19 Pandemic: Underlying Principles and Value of Projections. JAMA. 2020. doi:https://doi.org/10.1001/jama.2020.6585.

8. Gibaldi M, Perrier D. Pharmacokinetics. In: J. Swarbrick, ed. Drugs and the Pharmaceutical Sciences. New York, NY: Marcel Dekker, Volume 15, $2^{\text {nd }} E d, 1992$.

9. Tolles J, Luong T. Modeling Epidemics With Compartmental Models. JAMA. 2020. doi:https://doi.org/10.1001/jama.2020.8420.

10. Hale T, Webster S, Petherick A, Phillips T, Kira B. Oxford COVID-19 Government Response Tracker, Blavatnik School of Government, 2020. Available from: https://www.bsg.ox.ac.uk/research/research-projects/ coronavirus-government-response-tracker (29 July 2020, date last accessed).

11. López L, Rodó $\mathbf{X}$. The end of social confinement and COVID-19 reemergence risk. Nat Hum Behav. 2020. doi:https://doi.org/10.1038/ s41562-020-0908-8

12. Courtemanche C, Garuccio J, Le A, Pinkston J, Yelowitz A. Strong Social Distancing Measures In The United States Reduced The COVID-19 Growth Rate. Health Aff (Millwood). 2020;39:1237-1246. doi:10.1377/ hlthaff.2020.00608.

13. Castillo RC, Staguhn ED, Weston-Farber E. The effect of state-level stay-at-home orders on COVID-19 infection rates. Am J Infect Control. 2020. doi:https://doi.org/10.1016/j.ajic.2020.05.017.

14. Lyu W, Wehby GL. Comparison of Estimated Rates of Coronavirus Disease 2019 (COVID-19) in Border Counties in Iowa Without a Stayat-Home Order and Border Counties in Illinois With a Stay-at-Home Order. JAMA Netw Open. 2020;3:e2011102.

15. Sen S, Karaca-Mandic P, Georgiou A. Association of Stay-at-Home Orders With COVID-19 Hospitalizations in 4 States. JAMA. 2020;323:2522-2524.

Publisher's Note: Springer Nature remains neutral with regard to jurisdictional claims in published maps and institutional affiliations. 